\title{
IV \\ DE CODICIBUS LIBRI DE MIXTIONE
}

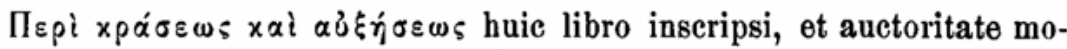
tus codicis $\mathrm{A}$, de quo infra disseram, et argumento libelli in quo repl

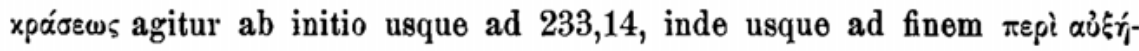

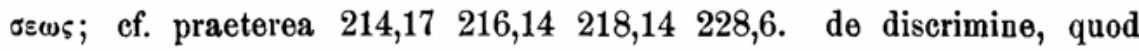
inter $x \rho \tilde{a} \sigma \iota v$ et $\mu \tilde{\xi} \xi \nu$ intercedit, videas $228,26 \mathrm{sq}$., unde liquet Parisini $\mathrm{R}$ et Aldinae') rubrum $\pi \varepsilon \rho i \mu^{\prime}(\xi \varepsilon \omega \varsigma$ falsum esse. tertium titulum Themistius praebet (Paraph. Phys. Ausc. 256,21) $\pi \varepsilon p i \times p \alpha ́ \sigma \varepsilon \omega \nu$, quo magis placet libris traditus. sed certo de hac re non licet diiudicare, quia non integer liber servatus est.

Codicum qui eum continent novi hos:

A Venetus Marcianus 257, in $4^{\circ}$ chartaceus fol. 283 saeculi circiter XII secundum Zanetti p. 127; rectius Morelli p. 144 saec. XIV eum ad-

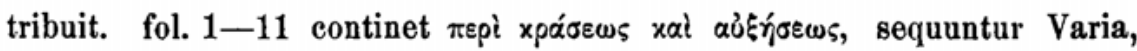
quorum catalogum a Zanettio confectum Morellius correxit l. c. permultis compendiis parumque diligenter scriptus est, prima pagina temporis iniuriis attrita lectu difficillima est. bis ipse contuli 1881 et 1887.

Qui sequuntur codices saeculi sunt XV vel XVI

B Taurinensis 94 fol. 84 inter varia excerpta mathematica et philosopha hune libellum continet fol. 43-55. contuli ipse. cf. Pasini catalogum I, 184.

R Parisinus 1848 continet f. $136-147$ nostrum libellum (contulit Elter)

S Parisinus 2540 f. 93-138 (contulit Elter)

P Parisinus 2028 f. 107-131 (contuli ipse. omnes tres libri Parisini Ioannis Francisci Asulani erant, ut ipse in primo folio adnotavit)

3) Ex Aldina autem videtur in Parisinum $R$ titulus venisse. nam in boc alia manu additum est testante Eltero. 
Vaticanus 1302 f. $203-218$ (contuli ipse)

Escorialensis X,I, 11 (cf. Miller 350) f. 64-79 (initium contulit L Bethe).

Accedit Aldina editio anni 1527 Philoponei commentarii ad Aristo- a telis De generatione et corruptione et Alexandri ad Meteorologica, quorum in calce impressus est hic liber sub titulo $\pi s p i \mu^{\prime} \xi \varepsilon \omega_{5}$.

Discedunt hi libri in tres classes, quorum primae unus testis est Venetus A, altera formatur his quinque BSCPL, tertia Parisino $\mathrm{R}$ et Aldina. hanc autem ex Parisino $\mathrm{R}$ vel gemino huius libri provenisse lacuna testatur 223,34 et adnotationis meae quaevis pagina.

BCPSL eodem originis vinculo contineri iterum et omissiones et menda demonstrant his quinque communia. conferas 213,21 214,28 $215,13218,20219,2223,16224,3 \quad 225,16.22 \quad 229,34238,14$ etc. alia

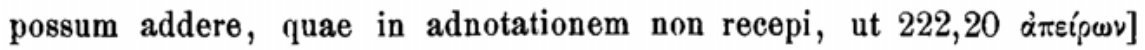

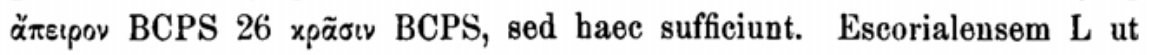
confidenter his adnumerem, etsi pars eius tantum mihi nota est, movet

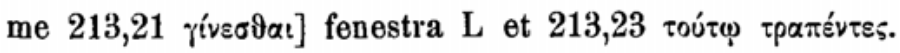

Nunc si quaerimus, quibus affinitatis gradibus hi libri distineantur, ${ }_{\mathrm{A}}^{\text {Arcietypus }}$ primum veri simillimum est patrem esse A codicum BCPS. nam si 213,

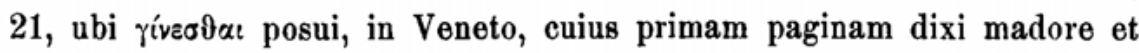
frictione laesam esse, huius verbi intermortua vix vestigia deteguntur, fenestra in BL hiat, cum CPS a verbo $\tau \dot{\alpha} \lambda \lambda \alpha$ sine lacunae indicio ad $\varepsilon \varphi^{\prime}$ transcurrant, B et L religiose, CPS neglegenter codicem A repraesentare videmus. accedit, quod et 218,5 et 229,34 idem suadent. quod si verum est, porro sequitur ex his locis

$235,4 \mu \tilde{\nu} s$ ex corr. ARa: om. BSP: fenestra C

238,14 Evaipwv ARa: fenestra BCPS

non ex ipso Veneto singulos descriptos esse, sed ex eius apographo nescio qua causa duobus his locis mutilato. praeterea pauci et levissimi errores in hoc libro correcti erant (cf. 215,13 218,33. 34 221,17. 29 (nam

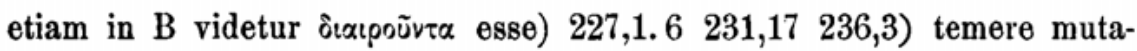

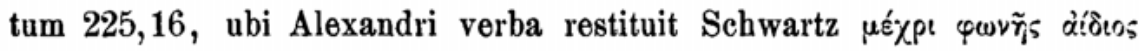
à̉ंoĩs, quod excepta una littera servavit $\mathrm{A}(\mu . \varphi$. aiōoĩos aj̉.), illi autem

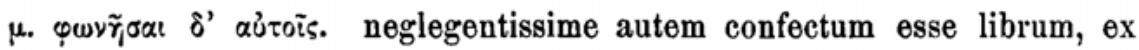
quo fluxerunt BCPS, pleraque vitia indicant filiis communia, quorum partem supra proposui. accedunt singulorum delicta, quae taedet singillatim proponere. hoc tantum ex accurata omnium collatione commemorabo, minime inter hos peccare Taurinensem, plurima et fere incre- 
dibilia Parisinum S. raro in singulis inveniuntur lectiones consideratione dignae, ut 215,29. $30223,1422 \bar{\imath}, 5.21229,2.3231,13$. levia sunt et ex parte videntur ad Parisinum $\mathrm{R}$ redire.

$\mathrm{A}^{2} \quad$ Neque multa adscripsit altera Veneti manus neque quae adscripserit, certo mibi licuit circumscribere. videntur autem primae manus correcturae esse eae, quibuscum consentiunt BCPS, ut 216,26. 31 235,2-3. contra iure mibi videor correctori aetatis minoris tribuere ea, quae con-

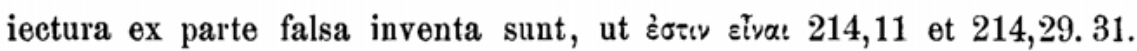
secutus sum eam 217,1 232,21. alia ex Parisino $R$ recte desumpsit: 214,27 219,2 233,28 235,6 236,26.

R Restat igitur, ut de Parisino $R$ iudicem. non pendet ex fonte codicum BCPS, nam 213,21 235,4 238,14 non est lacunosus. cum A autem hic quoque mire consentit. ita religiose compendium servavit, quod etiam in A exstat pro ú $\lambda$ ıxaĩs neglegenter formatum $(223,16)$; hoc BCPS falso solverunt in ítxais, a ut non intellectum omisit vacuo relicto. genuinum praebet $R$, ubi A corruptus est, non saepe quidem, sed praebet unus 213,9 216,20 228,9 237,28.31; accedunt ei loci, ubi $\mathrm{A}^{2}$ dixi ex $\mathbf{R}$ Venetum correxisse. sed obstat his virtutibus multo maior pars depravatarum lectionum, quas ex adnotatione nolo hic repetere. qua re verisimilius est, Parisinum quoque $R$ apographum quam fratrem esse Veneti. certe hic fundamentum recensionis debet esse, quem in apparatu cum Parisino $R$ integrum proposui ex BCPS graviora tantum adnotans. - Denique moneo lacunam in archetypo indicatam fuisse 229,21 .

Venimus ad veteres versiones latinas, quarum duas novi CaninII Anglariensis, quae primum prodiit V'enetiis $1555^{1}$ ) (editione usus sum anni 1559), et Iacobi Scheckir (Tubingae 1540). neutram in adnotationem criticam recepi. nam Caninium sive Aldina sive gemello codicis $\mathrm{R}$ usum esse demonstrat lacuna, quae 223,34 his cum Caninii interpretatione communis est. item 225,16 in verbis interpretis "cum vero talis sit, necessario alterutrum ipsis duntaxat dicendum erit" cognoseitur Pa-

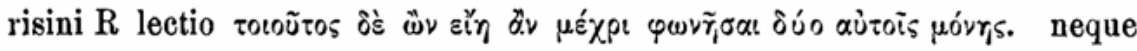
tacebo in media parte huius versionis magnam hiare lacunam. desunt

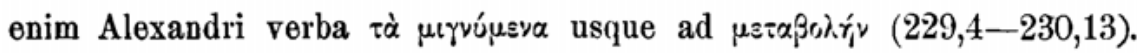
quae utrum in officina perierint latina an ad graecum exemplar mutilum referendum sit quod desunt, nescio, sed memorabile est post magnum

1) Cf. Hoffmann Lex. Bibl. I, 111. 
hiatum sequi apud Caninium eius enuntiati abruptum membrum per se sensu cassum, quocum ad verbum consentit initium fol. $145^{r}$ graecae Aldinae.

Etiam Schickirs ex Aldina pendet, quamquam quominus accuratius hoc persequar obstat neglegentissima et semibarbara huius versionis natura. quae quanta oscitantia conseripta sit, duo liceat exempla proponere.

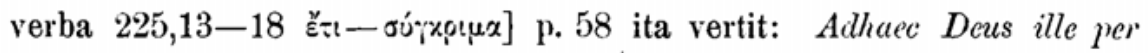
omnia permeans, materia ipsa tempore esset posterior, siquidem materiatum corpus omne, materia ipsa posterius est, Deus autem tale corpus est, quapropter duo principia non erunt, quae oratio eorum prae se fért, sed revera tantum unum erit, atque ille Deus procreatus ex materia aut elementum

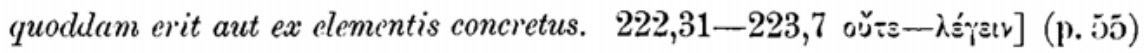
atqui forma nulla separatim esse potest, absque co in quo defixa est, ipsumque lumen nisi in pellucido separatim nemo intueri potest, neque anima sine corpore separatim consistit, et ut uno verbo dicam, quid absurdius cogitari potest, quam materiam cum sua forma misceri. quam rursus ridiculum illud ete.

Edidit libellum nostratium unus Ideler in Aristotelis Meteorologicorum vol. II p. 589-624 Lipsiae 1836, si editionis nomine oruare volumus Aldinae apographum neglegentissime (desunt enim non solum verba, sed tota enuntiata) confectum, in quo sanata quidem sunt pauca, sed multa mutata, quae vir doctissimus, si rem iterum pensitasset, ipse removisset.

Multo plura debeo Ottoni Apelt, qui doctissima commentatione inserta Philologi volumini XLV p. 82 sq. libellum nostrum persecutus alia feliciter emendavit aliis prudenter medelam praeparavit.

Habes subsidia, quibus usus sum in singulis Alexandri scriptis. nondum vero de auxilio dixi, quod milhi per omnia aeque paratum fuit iucundissimum, Hermanini Diels et Edvardi Schwartz amicorum. quorum opera factum est, ut viam emenso non tam molestiarum, quibus non caruit iter, surgat recordatio, quam laetissima laborum communionis memoria. qui viri doctissimi, dum in corrigendis typothetae erroribus me adiuvant, quantum studium emendandis ipsius Alexandri vel difficillimis librorum partibus impertiverint, mecum admirabuntur legentes sentientque, quid debeat baec editio illorum acumini. Vsenerr's autem, qua est erga me benevolentia, gratissima etian boc volumen ornare dignatus est symbola, quam invenient legentes in Addendis, ubi ea descripsi, quae in exemplo quo utitur Vsenerus Quaestionum Spengelianarum adnotavit. 